\title{
Identification of HZF1 as a novel target gene of the MEF2 transcription factor
}

\author{
XI LIU, EN-ZE JIN, JI-XIN ZHI and XUE-QI LI \\ Department of Cardiology, The Fourth Affiliated Hospital of Harbin \\ Medical University, Harbin, Heilongjiang 150001, P.R. China \\ Received December 28, 2010; Accepted March 21, 2011
}

DOI: $10.3892 / \mathrm{mmr} .2011 .465$

\begin{abstract}
The MADS box-containing transcription factors MEF2A-D regulate the expression of most skeletal and cardiac muscle structural genes. Recently, a zinc finger protein called HZF1 was identified as a transcription factor, and found to be highly expressed in cardiac muscle. By screening the transcription regulatory region of HZF1, we found that myocyte enhancer factor 2 (MEF2) potentially recognizes and binds the regulatory region of the HZF1 gene. We also found that the knockdown of MEF2A endogenous expression in human aortic smooth muscle cells decreases the expression of HZF1, while the enforced expression of MEF2A in turn promotes the expression of HZF1. Furthermore, we employed the luciferase reporter system and chromatin immunoprecipitation (ChIP) to demonstrate that MEF2A does in fact bind the regulatory region of $\mathrm{HZF} 1$, which suggests that $\mathrm{HZF1}$ is the direct target of MEF2A. Based on the fact that MEF2 proteins function as essential regulators of cardiac development and as important pathological factors for certain cardiac diseases, our finding that MEF2 positively regulates the expression of HZF1 may contribute to further research investigating the role of the zinc finger protein HZF1 in cardiac development and disease.
\end{abstract}

\section{Introduction}

The myocyte enhancer factor 2 (MEF2) group was first identified by Olson and colleagues in 1989 from differentiated myotubes (1). There are four homologous gene products in the MEF2 family, which are referred to as MEF2A-D. These transcription factors have been identified in mammalian cells, and MEF2-like sequences have been discovered in yeast (2). MEF2 proteins belong to the MADS-box family of transcription factors. The MEF2 DNA-binding domain is

Correspondence to: Professor Xue-Qi Li, Department of Cardiology, The Fourth Affiliated Hospital of Harbin Medical University, Harbin, Heilongjiang 150001, P.R. China

E-mail: zxslixueqi@hotmail.com

Key words: zinc finger protein gene, myocyte enhancer factor 2, transcription factor, cardiac development, cardiac dilation
56 amino acids in length and is known as the MADS box. This domain is flanked by a shorter region that contributes to dimerization and facilitates interaction with other proteins. The MADS box is highly conserved within MEF2 proteins and binds readily with the A/T-rich DNA consensus sequence $\left[\mathrm{CTA}(\mathrm{A} / \mathrm{T})_{4} \mathrm{TAA} / \mathrm{G}\right]$ present in the $5^{\prime}$ transcriptional regulatory regions of most skeletal and cardiac muscle structural genes characterized to date $(3,4)$. Other regions of the proteins encode transactivation domains (4). Besides their regulatory function in skeletal and cardiac muscle, MEF2A-D genes also possess regulatory function in immune and neuronal cells (5-8).

The zinc finger protein gene HZF1 (ZNF16) was first identified by Han et al in 2006 through screening of a human bone marrow cDNA library (9). The full reading frame of the HZF1 gene contains a 670 amino-acid peptide. The C-terminal of this peptide contains 17 uninterrupted zinc fingers, including 15 typical and 2 atypical $\mathrm{C} 2 \mathrm{H} 2$ zinc finger motifs and TGEKPYE repeats between every two zinc fingers (9). Recently, Minjie et al characterized three nuclear location signals within the zinc finger region of the HZF1 C-terminal and demonstrated that $\mathrm{HZF} 1$ is a transcriptional activator by investigating the role of the non-zinc finger region of the HZF1 N-terminal (10). However, little is known regarding the targeting of specific genes by HZF1 and its physiological functions, except that HZF1 seems to affect erythroid and megakaryocytic hematopoiesis (9).

In this study, we observed that the enforced expression of MEF2A promotes the expression of HZF1, while the knockdown of MEF2A decreases the expression of HZF1. Furthermore, the results of a luciferase reporter assay and chromatin immunoprecipitation (ChIP) showed that MEF2A binds the regulatory region of HZF1. Taken together, this shows that HZF1 is a novel direct target gene of MEF2.

\section{Materials and methods}

Cell lines and culture. The 293T cell line was purchased from the American type culture collection (ATCC) and grown in Dulbecco's modified Eagle's medium (DMEM, Gibico BRL) containing $10 \%$ (v/v) fetal bovine serum (FBS, Gibico BRL), penicillin $(100 \mathrm{U} / \mathrm{ml})$, streptomycin $(100 \mu \mathrm{g} / \mathrm{ml})$ and $2 \mathrm{mM}$ L-glutamine. Human aortic smooth muscle cells (HAOSMC) were purchased from Cell Applications Inc. and cultured in 
smooth muscle cell growth medium (Cell Applications Inc.). Both cell types were cultured in a humidified atmosphere of $95 \%$ air and $5 \% \mathrm{CO}_{2}$ at $37^{\circ} \mathrm{C}$.

RNA isolation and reverse transcription. Total RNA was extracted from the cells and skeletal muscle tissue using Trizol reagent (Invitrogen, USA) according to the manufacturer's instructions. The total RNA was quantified by measuring the absorbance at $260 \mathrm{~nm}$. The cDNA was synthesized by M-MLV reverse transcriptase (Invitrogen) from $2 \mu \mathrm{g}$ of total RNA with the primer Oligo $(\mathrm{dT})_{18}$.

Plasmid construction and transfection. The ORF fragment of MEF2A was amplified by PCR with the primers 5'-CTCGAG AAATGGGGCGGAAGAAAATAC-3' and 5'-GAATTCGCT TGGAAGCCTTAGGTCAC-3', and was then inserted into a $\mathrm{T}$ vector. After being sequenced, the amplified fragment was digested with $\mathrm{XbaI}$ (NEB, Ipswich, MA, USA) and EcoRI (NEB) followed by subcloning in-frame into the corresponding restriction site of the expression vector pcDNA-FLAG.

The recombinant plasmid pcDNA-FLAG-MEF2A and the control plasmid pcDNA-FLAG were transfected into 293T cells with Lipo2000 (Invitrogen) according to the manufacturer's instructions. Transfection was performed using a mixture of $2 \mu \mathrm{g}$ of recombinant expression plasmid and $5 \mu \mathrm{l}$ of Lipo2000, or $15 \mu \mathrm{g}$ plasmid and $30 \mu \mathrm{l}$ of Lipo2000 for a 6-well plate and $10 \mathrm{~cm}$ dish, respectively. Cells were harvested after $24 \mathrm{~h}$ of transfection in all cases.

Quantitative RT-PCR. Quantitative RT-PCR was carried out in a Bio-Rad real-time PCR system (Bio-Rad, USA) using a SYBR master mix kit (Roche, USA) according to the manufacturer's instructions. The comparative $\mathrm{Ct}$ method was used to quantify target genes relative to GAPDH (as the endogenous control). For each individual analysis, one of the samples was designated as the calibrator and given a relative value of 1.0. All quantities were expressed as $\mathrm{n}$-fold relative to the calibrator. Real-time PCR primers are listed in Table I.

$R N A$ interference. One day before transfection, $1 \times 10^{5}$ human aortic smooth muscle cells were plated in a 6-well plate. For the transfection, $5 \mu$ l Lipofectamine ${ }^{\mathrm{TM}} 2000$ was diluted in $245 \mu \mathrm{l}$ Opti-MEM and incubated for $5 \mathrm{~min}$ at room temperature. Then, 100 pmol of either MEF2A siRNA oligo (HSS106436, Invitrogen) or siRNA negative control (12935-330, Invitrogen) was mixed with $250 \mu$ l Opti-MEM. The diluted Lipofectamine 2000 was then combined with the diluted oligomer and incubated for $15 \mathrm{~min}$ at room temperature to allow complex formation to occur. The oligomer-Lipofectamine 2000 complexes were added to each well containing cells and medium and mixed gently by rocking the plate back and forth. The cells were incubated at $37^{\circ} \mathrm{C}$ in a $5 \% \mathrm{CO}_{2}$ incubator for $36 \mathrm{~h}$ prior to detection of the expression of the genes of interest.

Western blot assay. The total proteins were extracted from the cells using ice-cold lysis buffer (Sigma, USA). After extraction, $25 \mu \mathrm{g}$ of the protein samples were separated on a $10 \%$ SDS-PAGE gel and then transferred to polyvinylidene difluoride membranes (Amersham). The membrane was incubated
Table 1 Primers used in this study

Reporter plasmid constructs

Site 1+2 F: 5'-GCACGCGTGATAATAGATGTTGGCGTGG-3' R: 5'-GCCTCGAGGATTGGTGACATTTTGGC-3'

Site 1 F: 5'-GCACGCGTGAGATGTTGGCGTGGATGT-3' R: 5'-GCCTCGAGTGATTGATGGGCATTTGG-3'

Site 2 F: 5'-GCACGCGTGGGGAAAGTGCAGGAAGG-3' R: 5'-GCCTCGAGGGGCATCTGGCAGAAGAA-3'

qRT-PCR primers

GAPDH F: 5'-ATGGGGAAGGTGAAGGTCG-3'

R: 5'-GGGGTCATTGATGGCAACAATA-3'

HZF1

F: 5'-AAATCCTGACCTTATTCAGCGTC-3'

R: 5'-CCTAGAAAAGTCTGAGTGCCCTC-3'

MEF2 F: 5'-ATGCCATCAGTGAATCAAAGGAT-3'

R: 5'-CTGGTAAAGTAGGAGTTGCTACG-3'

with primary antibodies after blocking with 5\% degrease milk powder. After incubation with the primary antibody, the membrane was washed and incubated with peroxidaselinked secondary antibody $\operatorname{IgG}$ and then washed again. The membrane was then exposed to ECL hyper film (Amersham).

The primary antibodies used were rabbit monoclonal antibody to FLAG (Sigma), rabbit polyclonal antibody to human HZF1 (Abcam, USA) and mouse monoclonal antibody to GAPDH (Abcam). The secondary antibodies used were goat anti-rabbit IgG and goat anti-mouse IgG, and were purchased from Santa Cruz.

Bioinformatics analysis. To detect if MEF2 was directly binding to and regulating HZF1, we scanned the 2000 base pair upstream regulatory region of the HZF1 gene with the transcription factor binding sites prediction database TESS (http://www.cbil.upenn.edu/cgi-bin/tess/tess), TFSEARCH (http://www.cbrc.jp/research/db/TFSEARCH.html) and MOTIF (http://motif.genome.jp/) $(11,12)$.

Luciferase reporter constructs and luciferase assay. Promoters of HZF1 were amplified by PCR from human genomic DNA. PCR primers are listed in Table I. The PCR products were isolated and cloned into the MluI and XhoI (NEB) digested pGL3 basic vector (Promega) using T4 DNA ligase (Promega). All constructs were confirmed by sequencing. For the luciferase assay, $4 \times 10^{4} 293 \mathrm{~T}$ cells per well were plated in a 24-well plate overnight. Then, $250 \mathrm{ng}$ reporter plasmid was co-transfected into the $293 \mathrm{~T}$ cells with $740 \mathrm{ng}$ pcDNA-FLAG-MEF2A and $10 \mathrm{ng}$ internal control plasmid pRL-TK (Promega) using $3 \mu$ l Lipofectamine 2000 (Invitrogen). Twenty four hours later, cells were harvested and lysed with passive lysis buffer (Promega). Luciferase activity was measured using a dual luciferase reporter assay (Promega). Firefly luciferase activity was measured and normalized to the control Renilla luciferase level, and the luciferase activity of each construct was compared with that of the promoterless pGL3 basic vector. 

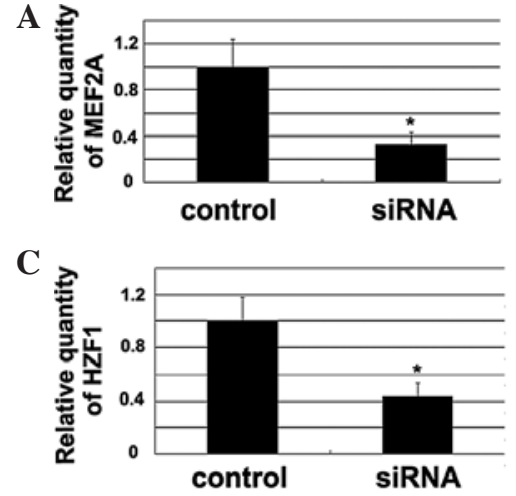

A

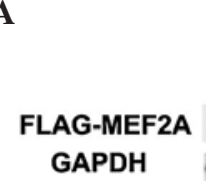

D

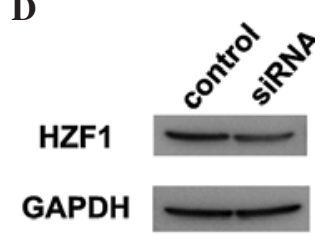

Figure 1. MEF2A knockdown down-regulates HZF1 expression. (A and B) Detection of the knockdown effect of MEF2A siRNA. MEF2A siRNA oligo and siRNA negative control were transfected into HAOSMC, and the expression change of MEF2A was examined $36 \mathrm{~h}$ after transfection using (A) quantitative RT-PCR and (B) Western blotting. The results suggest that MEF2A siRNA oligo successfully knocked down the endogenous expression of MEF2A at both the mRNA level $(\mathrm{P}<0.05)$ and at the protein level. $(\mathrm{C}-\mathrm{D})$ Detection of the expression change of HZF1 corresponding to the knockdown of MEF2A. The knockdown of MEF2A decreased the expression of HZF1 as shown by $(\mathrm{C})$ quantitative RT-PCR $(\mathrm{P}<0.05)$ and $(\mathrm{D})$ Western blotting.

Chromatin immunoprecipitation assay. The ChIP assay was performed as previously described (13) with some modifications. 293T cells were first transfected with either pcDNA-FLAG-MEF2A or pcDNA-FLAG, and then crosslinked with formaldehyde before being lysed. Subsequently, the cross-linked DNA was sheared by sonication to a mean average length of $500 \mathrm{bp}$ for conventional PCR analysis and verified by agarose gel electrophoresis. The chromatin fraction was immunoprecipitated overnight at $4^{\circ} \mathrm{C}$ using anti-FLAG. PCR amplification was performed in a total volume of $25 \mu \mathrm{l}$ with specific primers (forward, ACCTGGATGGGATTGGAGAC; reverse, AAGCAGCAAAGCATTCAAGATG).

\section{Results}

MEF2A knockdown down-regulated HZF1 expression. Small interference RNA (siRNA) were employed to knock down the expression of MEF2A and to determine its effect on the expression of HZF1. The MEF2A siRNA oligo and siRNA negative control were transfected into human aortic smooth muscle cells (HAOSMC) and the expression change of MEF2A was examined at $36 \mathrm{~h}$ post-transfection. The results showed that MEF2A siRNA efficiently knocked down the expression of MEF2A at both the mRNA and protein levels (Fig. 1A and B). Additionally, the expression change of HZF1 corresponding to the knockdown of MEF2A was detected, and the results of quantitative real-time PCR and Western blotting revealed that the knockdown of MEF2A decreased the expression of HZF1 (Fig. 1C and D).

MEF2A overexpression promotes HZF1 expression. The effect of MEF2A overexpression on the expression of HZF1 was observed. Either the recombinant plasmid pcDNAFLAG-MEF2A or the control plasmid pcDNA-FLAG were transfected into HAOSMC cells. The cells were harvested and lysed at $48 \mathrm{~h}$ post-transfection. Western blotting was performed to detect the expression of MEF2A in HAOSMC cells with

the FLAG antibody. As shown in Fig. 2A, the expression of fused protein FLAG-MEF2A was detected in pcDNAFLAG-MEF2A-transfected HAOSMC cells compared to pcDNA-FLAG-transfected cells, which confirmed the overexpression of the transcription factor MEF2A in HAOSMC cells. The expression of HZF1 at the transcriptional and translational levels was detected and compared in MEF2A overexpressed cells and control cells using real-time PCR and Western blotting. The results demonstrated that the HZF1 mRNA level in MEF2A overexpressed HAOSMC cells was 8-fold higher than in the control cells (Fig. 2B). Additionally, the level of HZF1 protein was significantly higher in MEF2A overexpressed cells as compared to the control cells (Fig. 2C). To eliminate the influence of the cell lines, MEF2A was expressed ectopically in $293 \mathrm{~T}$ cells, which under normal circumstances have very low expression of MEF2A (data not shown). The ectopic expression of MEF2A in 293T cells was found to promote the expression of HZF1 (Fig. 2D). These results suggest that the overexpression of MEF2A promotes the expression of HZF1.

HZF1 is the direct target of MEF2A. The results demonstrated that MEF2A positively regulates the expression of HZF1. However, it cannot be concluded from these results alone that HZF1 is a direct target of MEF2A, since it is possible that MEF2A indirectly affects the expression of HZF1 by way of regulating intermediate genes. Using bioinformatics analysis, two potential fragments (-1860 to -1849 and -1184 to -1174$)$ were found within the upstream regulatory region of $\mathrm{HZF} 1$ that correspond to the consensus sequence of MEF2 for recognition and binding. We cloned a series of fragments containing potential binding sites for MEF2A within the HZF1 promoter region (Fig. 3A) and installed them into a pGL3 basic vector, then co-transfected them with pcDNA-FLAG-MEF2A. The ectopic expression of MEF2A in 293T cells enhanced the luciferase activity of the reporters for site 2 ( -1184 to -1173$)$ by at least 7.5-fold as compared to the control (Fig. 3B). MEF2A 
A

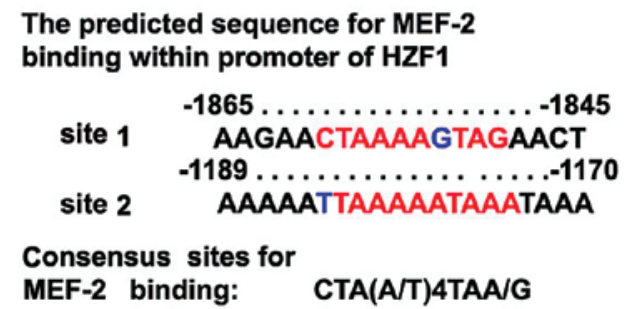

B

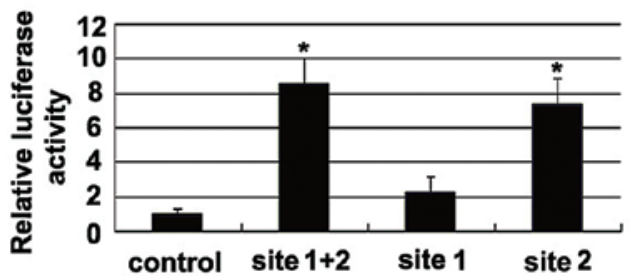

Figure 3. Binding sites for MEF2A within the regulatory region of the HZF1 gene identified using a luciferase reporter assay. (A) The scheme for the MEF2A potential binding sites within the region upstream of the HZF1 transcription start site. (B) Evidence for the MEF2A binding site on the HZF1 gene using a luciferase activity assay. A series of fragments containing MEF2A potential binding sites within the HZF1 promoter region was cloned and installed into a pGL3 basic reporter vector and then co-transfected with pcDNA-FLAG-MEF2A into 293T cells. The luciferase activity relative to the control was used to reflect the interaction between MEF2A and its potential binding sites. The results suggested that a DNA fragment from -1860 to -1848 upstream of the $\mathrm{HZF} 1$ transcription start site contributes to the recognition by MEF2A $(\mathrm{P}<0.05)$.

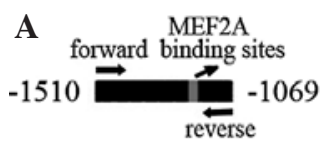

primers:

Forward:ACCTGGATGGGATTGGAGAC Reverse:AAGCAGCAAAGCATTCAAGATG

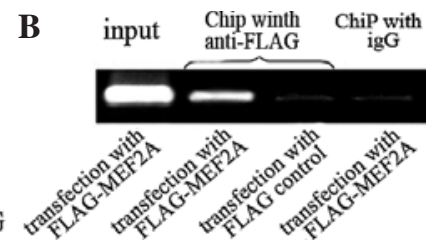

Figure 4. HZF1 is the direct target of MEF2A. (A-B) To confirm and validate the direct interaction between the transcription factor MEF2A and the HZF1 gene in vivo, specific primers were used to identify and quantify the DNA fragments containing MEF2A binding sites. (A) The input DNA and IgG pull-down DNA served as positive and negative controls, respectively. (B) The fragment containing the MEF2A binding sites extracted from FLAG-MEF2A-transfected cells had significantly greater binding by the FLAG-MEF2A antibody than the control cells.

also bound site 1 (-1860 to -1848), but with less efficiency, as demonstrated by the elevation in the luciferase activity of the reporter by MEF2A only at site 1 by approximately 2 -fold (Fig. 3B). These results suggest that MEF2A may regulate the expression of HZF1 mainly through binding the -1184 to -1173 DNA sequence upstream of the transcription start site.

ChIP was then employed to determine whether MEF2A directly binds sites located on the transcriptional regulatory region of HZF1. The identity and quantity of the DNA fragmentory isolated by immunoprecipitation was determined by PCR (Fig. 4A). The input DNA and IgG pull-down DNA served as a positive and negative control, respectively. The results demonstrated that the fragment containing the MEF2A binding site was enriched by the antibody to FLAG-MEF2A (Fig. 4B), which suggests that $\mathrm{MEF} 2 \mathrm{~A}$ binds the regulatory region of $\mathrm{HZF} 1$ in vivo, and that HZF1 is the direct target of MEF2A.

\section{Discussion}

In general, MEF2 genes are expressed in a broad range of cell types, including brain and neural crest cell derivatives as well as skeletal, cardiac and visceral muscle (14). While the HZF1 gene is also broadly expressed, its expression is particularly high in the brain, skeletal and cardiac muscle (9). Additionally, members of the MEF2 family have been implicated in the regulation of inducible gene expression in response to mitogen and stress stimulation (15). Similarly, our unpublished results have demonstrated that the over-expression of HZF1 promotes mitosis by activating the MAPK cell signaling pathway through an unknown mechanism. These common characteristics between HZF1 and MEF2 proteins imply that there may be a connection between the two genes. Bioinformatics analysis suggested that MEF2 regulates the expression of HZF1. Furthermore, our experiments demonstrated that HZF1 is a novel direct target gene for MEF2 proteins.

The ChIP assay is a very powerful method used to determine the location of DNA binding sites on the genome for a particular protein of interest. This technique presents a valuable picture of the protein-DNA interactions that occur inside the nucleus of living cells or tissues, in contrast to other approaches traditionally employed to answer the same question such as the electrophoretic mobility shift assay (EMSA) and the reporter gene assay, among others. In this study, we employed the ChIP assay and found that MEF2 directly regulates the expression of HZF1. It is important to note that the DNA sequence within the promoter region of HZF1 is not completely identical to the well-known MEF2 consensus binding sites; however, this phenomenon has previously been reported $(11,12)$. This finding suggests that the transcription factor MEF2 may regulate the expression of more genes than those currently known.

Our findings on the one hand provide insight into the function of MEF2 genes, and on the other suggest that it is possible to detect the physiological roles of HZF1 based on the known function of MEF2 genes. Thus, we summarized the main functions of MEF2 transcription factors in cardiac development and adult heart diseases to elucidate the role of HZF1 in the heart, which is one of the organs where HFZ1 is highly expressed.

The MEF2 family of transcription factors function as essential regulators of cardiac development. Loss of Mef $2 \mathrm{a}$ in mice leads to right ventricular dilation as a result of myofiber disorganization. The majority of Mef2a null mice die, possibly due to disruption of the cytoskeleton architecture or conduction system defects (16). The few Mef2a null mice that do survive into adulthood show a mitochondrial deficiency and possible conduction system defects (16). Additionally, targeted disruption of Mef2c in the mouse leads to early embryonic lethality due to the absence of the right ventricle (17). Transgenic mice that express a dominant-negative MEF2 mutation in the heart succumb to early postnatal lethality resulting from cardiomyocyte hypoplasia, ventricular wall thinning and chamber dilation (18). Together, these findings provide evidence that MEF2 protein is required for the proper differentiation and postnatal development of the heart.

There is some evidence to suggest that MEF2 is an important pathological factor involved in cardiac hypertrophy and 
cardiac dilation. The over-expression of MEF2A or MEF2C in the hearts of transgenic mice predisposes the heart to hypertrophic growth following pressure overload stimulation and simultaneously-induced ventricular chamber dilation and contractile dysfunction. Additionally, the isolated adult cardiomyocytes from MEF2A transgenic mice contain a predominant increase in length, and adenoviral-mediated over-expression of MEF2A or MEF2C in cultured neonatal cardiomyocytes induces sarcomere degeneration and focal elongation (19), which further suggests that MEF2 promotes cardiac dilation. Although the mechanism for MEF2 regulation of the hypertrophic growth of the heart is unclear, the enhancement of MEF2 DNA-binding activity may contribute to the regulation of pressure and volume overload-induced cardiac hypertrophy $(20,21)$. Finally, previous studies have shown that stretching of neonatal cardiomyocytes in culture also increased MEF2 DNA-binding activity $(22,23)$. In conclusion, data from overexpression experiments indicate that MEF2 is possibly involved in the pathologic hypertrophic response in cardiomyocytes.

Based on our findings in conjunction with research performed in other labs, we conclude that HZF1 is a novel direct target gene of the transcription factor MEF2. It is a reasonable and intriguing hypothesis that HZF1 may be a novel regulator of cardiac development and may also be involved in the pathologic mechanisms of certain heart diseases, including cardiac hypertrophy and cardiac dilation. These findings may contribute to future studies regarding the function of HZF1 in cardiac development and disease.

\section{References}

1. Gossett LA, Kelvin DJ, Sternberg EA and Olson EN: A new myocyte-specific enhancer-binding factor that recognizes a conserved element associated with multiple muscle-specific genes. Mol Cell Bi 9: 5022-5033, 1989.

2. Olson EN, Perry, M and Schulz RA: Regulation of muscle differentiation by the MEF2 family of MADS box transcription factor. Dev Biol 172: 2-14, 1995.

3. Black BL and Olson EN: Transcriptional control of muscle development by myocyte enhancer factor-2 (MEF2) proteins. Annu Rev Cell Dev Biol 14: 167-196, 1998.

4. Molkentin JD and Olson EN: Combinatorial control of muscle development by basic helix-loop-helix and MADS-box transcription factors. Proc Natl Acad Sci USA 93: 9366-9373, 1996.

5. Rao S, Karray S, Gackstetter ER and Koshland ME: Myocyte enhancer factor-related B-MEF2 is developmentally expressed in $\mathrm{B}$ cells and regulates the immunoglobulin $\mathbf{J}$ chain promoter. J Biol Chem 273: 26123-26129, 1998.

6. Han J, Jiang Y, Li Z, Kravchenko VV and Ulevitch RJ: Activation of the transcription factor MEF2C by the MAP kinase p38 in inflammation. Nature 386: 296-299, 1997.
7. Skerjanc IS and Wilton S: Myocyte enhancer factor $2 \mathrm{C}$ upregulates MASH-1 expression and induces neurogenesis in P19 cells. FEBS Lett 472: 53-56, 2000.

8. Lin Q, Schwarz J, Bucana C and Olson EN: Control of mouse cardiac morphogenesis and myogenesis by transcription factor MEF2C. Science 276: 1404-1407, 1997.

9. Peng H, Du ZW and Zhang JW: Identification and characterization of a novel zinc finger protein (HZF1) gene and its function in erythroid and megakaryocytic differentiation of K562 cells. Leukemia 20: 1109-1116, 2006.

10. Deng MJ, Li XB and Zhang JW: Identification of the transactivation domain and the nuclear location signals of human zinc finger protein HZF1 (ZNF16). Mol Biotechnol 44: 83-89, 2010.

11. Zhou R, Hu G, Gong AY and Chen XM: Binding of NF-kappaB p65 subunit to the promoter elements is involved in LPS-induced transactivation of miRNA genes in human biliary epithelial cells. Nucleic Acids Res 38: 3222-3232, 2010.

12. Zhou R, Hu G, Liu J, Gong AY, Drescher KM and Chen XM: NF-kappaB p65-dependent transactivation of miRNA genes following Cryptosporidium parvum infection stimulates epithelial cell immune responses. PLoS Pathog 5: Dec 4, 2009 (E-pub ahead of print).

13. Shang Y, Hu X, Di Renzo J, Lazar MA and Brown M: Cofactor dynamics and sufficiency in estrogen receptor-regulated transcription. Cell 103: 843-852, 2000.

14. Edmondson DG, Lyons GE, Martin JF and Olson EN: Mef2 gene expression marks the cardiac and skeletal muscle lineages during mouse embryogenesis. Development 120: 1251-1263, 1994.

15. Shore $P$ and Sharrocks AD: The MADS-box family of transcription factors. Eur J Biochem 229: 1-13, 1995.

16. Naya FJ, Black BL, Wu H, Bassel-Duby R, Richardson JA and Hill JA: Mitochondrial deficiency and cardiac sudden death in mice lacking the MEF2A transcription factor. Nat Med 8: 1303-1309, 2002

17. Bi W, Drake CJ and Schwarz JJ: The transcription factor MEF2C-null mouse exhibits complex vascular malformations and reduced cardiac expression of angiopoietin 1 and VEGF. Dev Biol 211: 255-267, 1999.

18. Kolodziejczyk SM, Wang L, Balazsi K, De Repentigny Y, Kothary R and Megeney LA: MEF2 is upregulated during cardiac hypertrophy and is required for normal post-natal growth of the myocardium. Curr Biol 9: 1203-1206, 1999.

19. Xu J, Gong NL, Bodi I, Aronow BJ, Backx PH and Molkentin JD: Myocyte enhancer factors $2 \mathrm{~A}$ and $2 \mathrm{C}$ induce dilated cardiomyopathy in transgenic mice. J Biol Chem 281: 9152-9162, 2006.

20. Molkentin JD and Markham BE: Myocyte-specific enhancerbinding factor (MEF-2) regulates alphacardiac myosin heavy chain gene expression in vitro and in vivo. J Biol Chem 268: 19512-19520, 1993

21. Nadruz WJ, Kobarg CB, Constancio SS, Corat PD and Franchini KG: Load-induced transcriptional activation of c-jun in rat myocardium: regulation by myocyte enhancer factor 2 . Circ Res 92: 243-251, 2003.

22. Shyu KG, Ko WH, Yang WS, Wang BW and Kuan P: Insulinlike growth factor-1 mediates stretch-induced upregulation of myostatin expression in neonatal rat cardiomyocytes. Cardiovasc Res 68: 405-414, 2005.

23. Nadruz WJ, Corat MA, Marin TM, Guimaräes Pereira GA and Franchini KG: Focal adhesion kinase mediates MEF2 and c-Jun activation by stretch: role in the activation of the cardiac hypertrophic genetic program. Cardiovasc Res 68: 87-97, 2005. 